\title{
Diffusion tensor magnetic resonance imaging: is it valuable in the detection of brain microstructural changes in patients having migraine without aura?
}

\author{
Saher E. Taman ${ }^{A, B, C, D, E, F}$, Wael H. Kamr ${ }^{A, B, C, E, F}$, Tamer M. Belal| ${ }^{B, C, D, F}$, Ahmed I. Tawfik ${ }^{A, B, C, E, F}$ \\ Faculty of Medicine, Mansoura University, Egypt
}

\section{Abstract}

Purpose: The aim of this study is to assess the diagnostic value of diffusion tensor magnetic resonance imaging (MRI) in the detection of brain microstructural changes in patients having migraine without aura.

Material and methods: Our prospective study included 33 patients having migraine without aura and 15 volunteers with matched age and sex, who underwent brain MRI with diffusion tensor imaging (DTI). The fractional anisotropy (FA) and mean diffusivity (MD) of selected grey and white matter regions on both sides were measured and correlated with the neurological clinical examination.

Results: Significant differences were detected in MD values in the thalamus, globus pallidus, and hippocampus head on the right side of patients versus controls. Also, significant differences of the FA values were detected in the thalamus, globus pallidus, and hippocampus head on the right side of patients versus controls. Regarding the FA values of the same regions on the left side, a significant difference in the FA value was detected only in the hippocampus head. There was a statistically significant difference in the FA values on both sides of the white matter of the frontal lobes, posterior limbs of the internal capsules, and cerebellar hemispheres in patients compared to controls. There was a statistically significant difference in MD values in the white matter of both frontal lobes, posterior limb of the right internal capsule, and both cerebellar hemispheres in patients compared to controls.

Conclusions: DTI can detect microstructural changes of the grey and white matter in patients having migraine without aura that could not be detected by conventional MRI.

Key words: brain, MRI, migraine, diffusion tensor.

\section{Introduction}

Migraine is a disorder in which recurrent headache occurs, which is pulsating in quality, moderate to severe in intensity, and unilateral in its location. It is usually aggravated by routine physical activity. It can be associated with nausea and/or phonophobia $[1,2]$. It has high prevalence, a high socioeconomic burden, and severely affects life quality. It was ranked as the sixth cause of disability worldwide and recorded among the top 10 causes of disability interfering with daily living activities [3-8]. It is generally believed that migraine is an abnormality in the brain function caused by a neurovascular disorder in which cortical spreading depression, neurogenic inflammation, and cranial vascular contractile dysfunction are shared in its pathophysiology. The characteristic headache results from dilatation of the extra-parenchymal blood vessels following the activation of the trigeminovascular system with subsequent release of multiple peptides leading to inflammation and vascular dilatation, resulting in

Correspondence address:

Wael H. Kamr, Faculty of Medicine, Mansoura University, Egypt, e-mail: dr.waelkamr@gmail.com

Authors' contribution:

A Study design · B Data collection · C Statistical analysis · D Data interpretation · E Manuscript preparation · F Literature search · G Funds collection 
the characteristic headache of either migraine with aura or migraine without aura [9-11].

\section{Imaging modalities}

The most powerful imaging modalities used in the diagnosis of central nervous system abnormalities is magnetic resonance imaging (MRI). In patients with migraine, routine MRI revealed normal results in most cases. In the remaining cases abnormal white matter hyperintensities could be seen on T2 and FLAIR (fluid attenuated inversion recovery) images. They are typically multiple and small, and punctate in the deep periventricular white matter and centrum semiovale. Recent studies done using 3 Tesla machines revealed increased T2 signal intensity of the cerebral cortex overlying the white matter abnormalities as well as in the brainstem [12-14]. Functional MRI provided more information by demonstration of cerebral physiological and morphological changes during attacks of migraine. Imaging using positron emission tomography (PET) showed sits of activity during migraine attacks, but it has limited anatomical resolution interfering with accurate detection of the exact structure involved during the attack [15].

\section{Diffusion tensor imaging basics}

Recent advancements in the technology of neuroimaging have provided more powerful methods helping us to understand the pathological mechanism of migraine. It can also help in identification of abnormal brain regions associated with migraine [2-8]. Diffusion-tensor imaging (DTI) is an excellent MRI technique for investigating brain parenchymal microstructural changes. This can be achieved through its power to assess the diffusion of water along and across white matter tracts [16]. The applications of DTI have been rapidly increasing in recent years because it is highly sensitive to minor changes at the microstructural and cellular levels. DTI images obtained by incorporating directionality into a diffusion-weighted measurement, allowing the extraction of the diffusion anisotropy characteristics of certain areas of the brain, thus giving details about their microstructural integrity. Fractional anisotropy (FA) and mean diffusivity (MD) are the 2 main parameters used in the quantification of directionality and amplitude of water diffusion [14].

\section{Material and methods}

\section{Patients}

Institutional review board permission was obtained for this prospective study carried out from June 2018 to February 2019. Informed consent was obtained from each patient and from controls before the MRI examination was done. The study included 33 patients ( 5 males and 28 females, mean age 35.03 years), which was defined as the patient group and 15 (age- and sex-matched) healthy volunteers ( 2 males and 13 females, mean age 35 years), which was defined as the control group. All patients were referred from the Neurology Clinic with a diagnosis of migraine without aura. The patients and controls underwent routine MRI study with DTI of the brain. The inclusion criteria were patients having migraine without aura and not having other clinically apparent disease by full neurological and general clinical examination. The exclusion criteria were those patients with claustrophobia and those having a prosthesis incompatible with MRI examination, such as a cardiac pacemaker and prosthetic cardiac valve. Patients and controls were pain free at the time of MRI examination, and patients were free of migraine for at least 2 days before the MRI exam, so the imaging was performed between migraine attacks, which is known as the interictal phase. Female patients were scanned in the mid-cycle. Initially the study included 35 patients and 16 healthy volunteers. Three were excluded because of abnormal MRI findings (2 patients had intracranial masses) and excessive movement during the examination (one volunteer). Finally, the study contained 33 patients and 15 volunteers.

\section{Clinical examination}

Full clinical and neurological examination was done for all patients $(n=33)$ and controls $(n=15)$ by a neurology consultant (BT) with 18 years' experience. All patients were screened for history of head trauma, psychiatric disorders, or acute pain disorders other than migraine, which, when present, excluded them from the study. The diagnosis of migraine was made using the International Classification of Headache Disorders (III) diagnostic criteria [17].

\section{Magnetic resonance imaging}

Brain MRI examination with DTI was done for all patients and controls. The MRI was carried out using a 1.5 Tesla scanner (Ingenia, Philips) using a Head Neck 20-channel coil. First, routine sequences were done as follows: T1WI $(\mathrm{TR} / \mathrm{TE}=620 / 20 \mathrm{~ms}), \mathrm{T} 2 \mathrm{WI}(\mathrm{TR} / \mathrm{TE}=5430 / 95 \mathrm{~ms})$, and FLAIR $(\mathrm{TR} / \mathrm{TE} / \mathrm{TI}=10500 / 120 / 2800 \mathrm{~ms})$ with matrix $=80 \times 80$, field of view $(\mathrm{FOV})=240 \times 220 \mathrm{~mm}$, and slice thickness $=5 \mathrm{~mm}$. Then DTI was done using a single-shot echo-planar imaging sequence $(\mathrm{TR} / \mathrm{TE}=3118 / 93 \mathrm{~ms})$ with sensitivity Encoding (SENSE). Then diffusion gradients were applied along 32 axes, using a b-value of 0 and $1000 \mathrm{~s} / \mathrm{mm}^{2}$. FOV $=240 \times 240 \mathrm{~mm}^{2}$ and data matrix $=92$ $\times 88$ were used, leading to voxel dimensions $2.43 \times 2.54$ $\times 2.5 \mathrm{~mm}$. Forty-eight slices were obtained, with a thickness of $2.5 \mathrm{~mm}$ and no gap, and the total scan time was 7-8 minutes.

\section{Image analysis}

The image analysis was done by a neuroradiologist with 18 years of experience (TS), who was blinded to the 

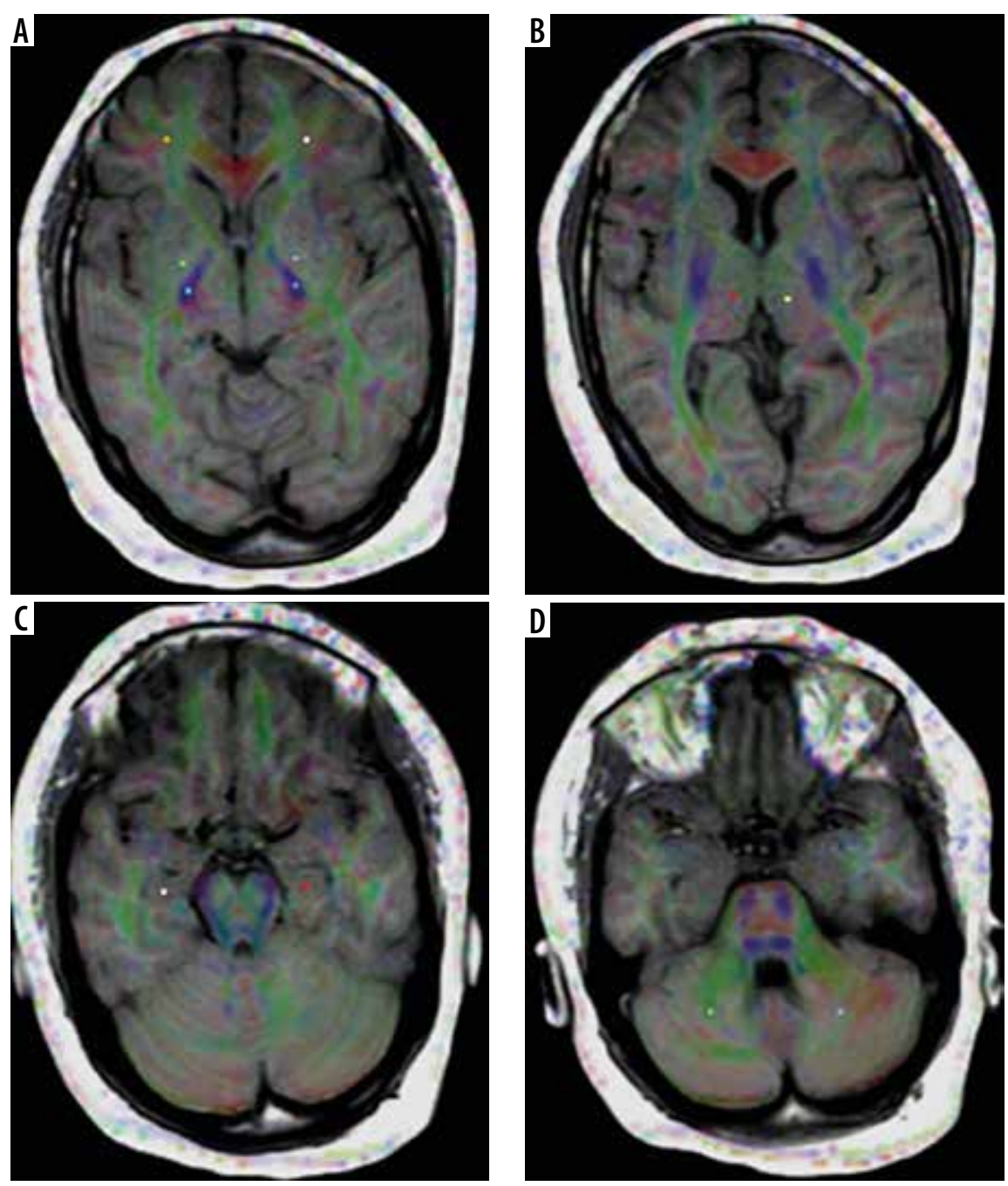

Figure 1. Receiver operating characteristic (ROI) placement for mean diffusivity and fractional anisotropy measurements at different regions of the brain. A) Frontal white matter, globus pallidus, and posterior limb of the internal capsule. B) Thalamus. C) Hippocampal head. D) (erebellar white matter

clinical data. First, the routine MR images were reviewed by the neuroradiologist, and they were excluded from the study if any abnormality was detected. The images were transferred to a workstation (extended MR Workspace 2.6.3.5, Philips medical systems Nederland B.V) supplied by the vendor. Measurements of MD and FA were obtained in 3 grey matter regions on each side (thalamus, globus pallidus, and hippocampus head) and 3 white matter regions on each side (frontal lobes, posterior limb of the internal capsule, and cerebellum). The total comprised 6 grey matter and 6 white matter regions. The readings were calculated and registered separately for each side (Figure 1).

\section{Statistical analysis}

Statistical analyses of this study were done using Statistical Package for Social Sciences (SPSS, Chicago, IL) version 20. Quantitative data were presented as mean and standard deviation (SD). Normally distributed data were compared between the 2 groups using the independent samples $t$-test. Data that violated the normality assump- tions were compared using the Mann-Whitney test. Probability $(p)$ values $<0.05$ were considered statistically significant. The receiver operating characteristic (ROC) curve was done to evaluate the diagnostic capability of the FA and MD in differentiating patients from controls. The area under the curve (AUC), accuracy, sensitivity, and specificity together with positive and negative predictive values were calculated.

\section{Results}

\section{Demographic}

The demographic criteria of the patients and controls are presented in Table 1. For the patient group $n=33$, males $n=5$, and females $n=28$. The mean age was $35.03 \pm 7.76$ years. Disease duration ranged from 1 to 24 years, mean approx. 10 years, the frequency of attacks was 2 to 6 times per month, mean approx. 4.5 times, side affected was right $n=11$, left $n=19$, and bilateral $n=3$. Last attack before MRI examination ranged from 0.6 to 4 weeks. The control group comprised $n=15$, males $n=2$, and females 
Table 1. Demographic data of patients and control groups

\begin{tabular}{|l|l|c|c|}
\hline \multicolumn{2}{|l|}{ Variables } & $\begin{array}{c}\text { Patients } \\
(n=33)\end{array}$ & $\begin{array}{c}\text { Controls } \\
(n=15)\end{array}$ \\
\hline \multirow{2}{*}{ Mean age } & $35.03 \pm 7.76$ & $35.00 \pm 5.57$ \\
\hline \multirow{2}{*}{ Gender } & Male & 5 & 2 \\
\cline { 2 - 4 } & Female & 28 & 13 \\
\hline \multicolumn{2}{|l|}{ Disease duration in years } & 10 & - \\
\hline \multicolumn{2}{|l|}{ Frequency of attacks per month } & 4.45 & - \\
\hline \multirow{2}{*}{ Side affected } & Right & 11 & - \\
\cline { 2 - 4 } & Left & 19 & - \\
\cline { 2 - 4 } & Bilateral & 3 & - \\
\hline
\end{tabular}

$n=13$. The mean age was $35.00 \pm 5.57$ years. All patients were right-handed.

\section{Routine magnetic resonance imaging}

On routine brain MRI examinations, no abnormality could be detected in any of the patient group and controls.

\section{Grey matter}

There were significantly increased MD values in the thalamus, globus pallidus, and hippocampus head on the right side of patients versus controls $(p=0.001,0.001$, and 0.001 , respectively). The measured MD values for the pa-

Table 2. Mean and standard deviation (minimum, maximum) of mean diffusivity (MD) and fractional anisotropy (FA) of the selected grey matter regions in patients and control groups

\begin{tabular}{|c|c|c|c|c|c|c|c|}
\hline \multirow[t]{2}{*}{ Variable } & \multirow[t]{2}{*}{ ROI site } & \multicolumn{2}{|c|}{$\begin{array}{c}\text { Patients }(n=33) \\
\text { Mean } \pm \text { SD (Min-Max) }\end{array}$} & \multicolumn{2}{|c|}{$\begin{array}{c}\text { Controls }(n=15) \\
\text { Mean } \pm \text { SD (Min-Max) }\end{array}$} & \multicolumn{2}{|c|}{$p$-values } \\
\hline & & Right & Left & Right & Left & Right & Left \\
\hline \multirow[t]{4}{*}{ MD } & Thalamus & $0.87 \pm 0.09$ & $0.76 \pm 0.16$ & $0.52 \pm 0.14$ & $0.82 \pm 0.02$ & 0.001 & 0.18 \\
\hline & Globus pallidus & $0.8 \pm 0.06$ & $0.75 \pm 0.03$ & $0.7 \pm 0.17$ & $0.75 \pm 0.01$ & 0.001 & 0.92 \\
\hline & Hippocampal head & $0.98 \pm 0.14$ & $0.95 \pm 0.25$ & $0.83 \pm 0.06$ & $0.88 \pm 0.07$ & 0.001 & 0.29 \\
\hline & Thalamus & $0.24 \pm 0.03$ & $0.24 \pm 0.05$ & $0.33 \pm 0.02$ & $0.27 \pm 0.06$ & 0.001 & 0.07 \\
\hline \multirow[t]{2}{*}{ FA } & Globus pallidus & $0.14 \pm 0.03$ & $0.17 \pm 0.04$ & $0.28 \pm 0.06$ & $0.16 \pm 0.04$ & 0.001 & 0.51 \\
\hline & Hippocampal head & $0.14 \pm 0.03$ & $0.13 \pm 0.03$ & $0.24 \pm 0.03$ & $0.19 \pm 0.04$ & 0.001 & 0.001 \\
\hline
\end{tabular}

Table 3. Results of receiver operating characteristic (ROC) curve of mean diffusivity (MD) of the selected grey matter regions with calculation of cut-off values, area under the curve (AUC), accuracy, sensitivity, and specificity in differentiating patients and controls

\begin{tabular}{|c|c|c|c|c|c|c|c|c|c|}
\hline \multicolumn{2}{|c|}{ Variable } & ROI site & Cut-off value & AUC & Sens. & Spec. & PPV & NPV & Accuracy \\
\hline \multirow{6}{*}{$\stackrel{\circ}{\Sigma}$} & \multirow{3}{*}{ '도 } & Thalamus & 0.73 & 0.978 & $97 \%$ & $86.7 \%$ & $94.1 \%$ & $92.9 \%$ & 93.8 \\
\hline & & Globus pallidus & 0.72 & 0.952 & $93.9 \%$ & $93.3 \%$ & $96.9 \%$ & $87.5 \%$ & $93.8 \%$ \\
\hline & & Hippocampal head & 0.89 & 0.814 & $72.7 \%$ & $86.7 \%$ & $92.3 \%$ & $59.1 \%$ & $77.1 \%$ \\
\hline & \multirow{3}{*}{ り } & Thalamus & 0.83 & 0.411 & $39.4 \%$ & $33.3 \%$ & $56.5 \%$ & $20 \%$ & $37.5 \%$ \\
\hline & & Globus pallidus & 0.75 & 0.413 & $42.4 \%$ & $60 \%$ & $70 \%$ & $32.1 \%$ & $47.9 \%$ \\
\hline & & Hippocampal head & 0.85 & 0.44 & $54.5 \%$ & $26.7 \%$ & $62.1 \%$ & $21.1 \%$ & $45.8 \%$ \\
\hline
\end{tabular}

Table 4. Results of receiver operating characteristic (ROC) curve of fractional anisotropy (FA) of the selected grey matter regions with calculation of cut-off values, area under the curve (AUC), accuracy, sensitivity, and specificity in differentiating patients and controls

\begin{tabular}{|c|c|c|c|c|c|c|c|c|c|}
\hline \multicolumn{2}{|c|}{ Variable } & ROI site & Cut-off value & AUC & Sens. & Spec. & PPV & NPV & Accuracy \\
\hline \multirow{6}{*}{ 壬 } & \multirow{3}{*}{ 도 } & Thalamus & 0.3 & 0.983 & $97 \%$ & $93.3 \%$ & $97 \%$ & $93.3 \%$ & $95.8 \%$ \\
\hline & & Globus pallidus & 0.2 & 0.984 & $93.9 \%$ & $73.3 \%$ & $88.6 \%$ & $84.6 \%$ & $87.5 \%$ \\
\hline & & Hippocampal head & 0.22 & 0.986 & $97 \%$ & $73.3 \%$ & $88 \%$ & $91.7 \%$ & $89.6 \%$ \\
\hline & \multirow{3}{*}{ • } & Thalamus & 0.26 & 0.691 & $72.7 \%$ & $53.3 \%$ & $77.1 \%$ & $47.1 \%$ & $66.7 \%$ \\
\hline & & Globus pallidus & 0.17 & 0.433 & $45.5 \%$ & $40 \%$ & $62.5 \%$ & $25 \%$ & $43.8 \%$ \\
\hline & & Hippocampal head & 0.17 & 0.895 & $84.8 \%$ & $80 \%$ & $90.3 \%$ & $70.6 \%$ & $83.3 \%$ \\
\hline
\end{tabular}


tients were $0.87 \pm 0.09,0.8 \pm 0.06$, and $0.98 \pm 0.14 \times 10^{-3}$ $\mathrm{mm}^{2} / \mathrm{s}$, respectively, while those of the controls were 0.52 $\pm 0.14,0.7 \pm 0.17$, and $0.83 \pm 0.06 \times 10^{-3} \mathrm{~mm}^{2} / \mathrm{s}$, respectively. Regarding the MD values of the same regions on the left side, the values were not statistically significant ( $p=0.18,0.92$, and 0.29 , respectively) in patients compared to controls (Table 2). In ROC curve analysis, the AUC of the MD values of the thalamus, globus pallidus, and hippocampus head on the right side used to differentiate patients from controls were $0.978,0.952$, and 0.814 , respectively, with the cut-off point to differentiate both groups at $0.73,0.72$, and $0.89 \times 10^{-3} \mathrm{~mm}^{2} / \mathrm{s}$, respectively. For the thalamus the sensitivity, specificity, and positive and negative predictive values were $97 \%, 86.7 \%, 94.1 \%$, and $92.9 \%$, respectively, with an accuracy of $93.8 \%$. For the globus pallidus the sensitivity, specificity, and positive and negative predictive values were $93.9 \%, 93.3 \%, 96.9 \%$, and $87.5 \%$, respectively, with an accuracy of $93.8 \%$. For the hippocampus head the sensitivity, specificity, and positive and negative predictive values were $72.7 \%, 86.7 \%, 92.3 \%$, and $59.1 \%$, respectively, with an accuracy of $77.1 \%$ (Table 3 ).

There were significantly decreased FA values in the thalamus, globus pallidus, and hippocampus head on the right side of patients versus controls $(p=0.001,0.001$, and 0.001 , respectively). The measured FA values for the patients were $0.24 \pm 0.03,0.14 \pm 0.03$, and $0.14 \pm 0.03$, respectively, while those of the controls were $0.33 \pm 0.02,0.28 \pm 0.06$, and $0.24 \pm 0.03$, respectively. Regarding the FA values of the same regions on the left side, a significant decrease in the FA value was detected only in the hippocampus head $(p=0.001)$. The measured FA value for the patients was $0.13 \pm 0.03$ while that of the controls was $0.19 \pm 0.04$. The FA values of the thalamus and globus pallidus on the left side were not statistically significant $(p=0.07$ and 0.51 , respectively) in patients compared to controls (Table 2).

In ROC curve analysis the AUC of the FA values of the thalamus, globus pallidus, and hippocampus head on the right side used to differentiate patients from controls were $0.983,0.984$, and 0.986 , respectively, and the cut-off points to differentiate both groups were $0.3,0.2$, and 0.22 , respectively. For the thalamus the sensitivity, specificity, and positive and negative predictive values were $97 \%$,
93.3\%, 97\%, and 93.3\%, respectively, with an accuracy of 95.8\%. For the globus pallidus the sensitivity, specificity, and positive and negative predictive values were $93.9 \%$, $73.3 \%, 88.6 \%$, and $84.6 \%$, respectively, with an accuracy of $87.5 \%$. For the hippocampus head the sensitivity, specificity, and positive and negative predictive values were $97 \%$, $73.3 \%, 88 \%$, and $91.7 \%$, respectively, with an accuracy of $89.6 \%$. On the left side of the hippocampus head the AUC of the FA value used to differentiate patients from controls was 0.895 and the cut-off point to differentiate both groups was 0.17 . Sensitivity, specificity, and positive and negative predictive values were $84.8 \%, 80 \%, 90.3$, and $70.6 \%$, respectively, with an accuracy of $83.3 \%$ (Table 4 ).

\section{White matter}

There was statistically significant lower FA on both sides of the white matter of the frontal lobes, posterior limbs of the internal capsules, and cerebellar hemispheres in patients compared to controls. On the right, $p=0.001$, 0.001 , and 0.001 , respectively, while on the left $p=0.001$, 0.005 , and 0.001 , respectively. The measured FA values of the patients on the right were $0.35 \pm 0.13,0.66 \pm 0.06$, and $0.29 \pm 0.08$, respectively, and on the left $0.34 \pm 0.09,0.57$ \pm 0.15 , and $0.32 \pm 0.15$, respectively, and for the controls, the measured FA values on the right were $0.62 \pm 0.1,0.73$ \pm 0.04 , and $0.47 \pm 0.09$, respectively, and on the left 0.46 $\pm 0.07,0.71 \pm 0.07$, and $0.52 \pm 0.12$, respectively (Table 5).

In ROC curve analysis, the AUC of the FA values on the right side were asfollows: frontal lobe $=0.931$, posterior limb of internal capsule $=0.82$, and cerebellum $=0.93$. On the left side: frontal lobe $=0.84$, posterior limb of internal capsule $=0.715$ and cerebellum $=0.843$. The cut-off points to differentiate both groups on the right were $0.56,0.71$, and 0.44 , respectively, while on the left they were $0.42,0.71$, and 0.47 , respectively. On the right side, for frontal lobe white matter, the sensitivity, specificity, and positive and negative predictive values were $93.9 \%, 80 \%, 91.2 \%$, and $85.7 \%$, respectively, with an accuracy of $89.5 \%$. For the posterior limb of the internal capsule, the sensitivity, specificity, and positive and negative predictive values were $75.8 \%$, $86.7 \%, 92.6 \%$, and $61.9 \%$, respectively, with an accuracy of

Table 5. Mean and standard deviation (minimum, maximum) of fractional anisotropy (FA) and mean diffusivity (MD) of the selected white matter regions

\begin{tabular}{|c|c|c|c|c|c|c|c|}
\hline \multirow[t]{2}{*}{ Variable } & \multirow[t]{2}{*}{ ROI site } & \multicolumn{2}{|c|}{$\begin{array}{c}\text { Patients }(n=33) \\
\text { Mean } \pm \text { SD (Min-Max) }\end{array}$} & \multicolumn{2}{|c|}{$\begin{array}{c}\text { Controls }(n=15) \\
\text { Mean } \pm \text { SD (Min-Max) }\end{array}$} & \multicolumn{2}{|c|}{$p$-value } \\
\hline & & Right & Left & Right & Left & Right & Left \\
\hline \multirow[t]{3}{*}{ MD } & Frontal white matter & $0.81 \pm 0.08$ & $0.83 \pm 0.06$ & $0.75 \pm 0.03$ & $0.75 \pm 0.02$ & 0.008 & 0.001 \\
\hline & Internal capsule & $0.75 \pm 0.04$ & $0.74 \pm 0.05$ & $0.68 \pm 0.05$ & $0.73 \pm 0.05$ & 0.001 & 0.68 \\
\hline & Cerebellar white matter & $0.81 \pm 0.08$ & $0.82 \pm 0.09$ & $0.68 \pm 0.04$ & $0.68 \pm 0.05$ & 0.001 & 0.001 \\
\hline \multirow[t]{3}{*}{ FA } & Frontal white matter & $0.35 \pm 0.13$ & $0.34 \pm 0.09$ & $0.62 \pm 0.1$ & $0.46 \pm 0.07$ & 0.001 & 0.001 \\
\hline & Internal capsule & $0.66 \pm 0.06$ & $0.57 \pm 0.15$ & $0.73 \pm 0.04$ & $0.71 \pm 0.07$ & 0.001 & 0.005 \\
\hline & Cerebellar white matter & $0.29 \pm 0.08$ & $0.32 \pm 0.15$ & $0.47 \pm 0.09$ & $0.52 \pm 0.12$ & 0.001 & 0.001 \\
\hline
\end{tabular}


$79.2 \%$. For the cerebellum, the sensitivity, specificity, and positive and negative predictive values were $93.9 \%, 86.7 \%$, 93.9\%, and $86.7 \%$, respectively, with an accuracy of $91.7 \%$. On the left side, for frontal lobe white matter, the sensitivity, specificity, and positive and negative predictive values were $78.8 \%, 66.7 \%, 83.9 \%$, and $58.8 \%$, respectively, with an accuracy of $75 \%$. For the posterior limb of the internal capsule, the sensitivity, specificity, and positive and negative predictive values were $69.7 \%, 60 \%, 79.3 \%$, and $47.4 \%$, respectively, with an accuracy of $66.7 \%$. For the cerebellum, the sensitivity, specificity, and positive and negative predictive values were $84.8 \%, 66.7 \%, 84.8 \%$, and $66.7 \%$, respectively, with an accuracy of $79.2 \%$ (Table 6 ).

There were statistically significant increased MD values in the white matter of both frontal lobes, posterior limb of the right internal capsule, and both cerebellar hemispheres in patients compared to controls. On the right-side frontal lobe, posterior limb of the internal capsule, and cerebellar hemisphere $p=0.008,0.001$, and 0.001 , respectively. The measured MD values for the patients were $0.81 \pm 0.08,0.75 \pm 0.04$, and $0.81 \pm 0.08 \times 10^{-3}$ $\mathrm{mm}^{2} / \mathrm{s}$, respectively while those of the controls were 0.75 $\pm 0.03,0.68 \pm 0.05$, and $0.68 \pm 0.04 \times 10^{-3} \mathrm{~mm}^{2} / \mathrm{s}$, respectively. On the left side frontal lobe and cerebellar hemisphere $p=0.001$ and 0.001 , respectively. The measured $\mathrm{MD}$ values for the patients were $0.83 \pm 0.06$ and $0.82 \pm$ $0.09^{\prime} 10^{-3} \mathrm{~mm}^{2} / \mathrm{s}$, respectively, while those of the controls were $0.75 \pm 0.02$ and $0.68 \pm 0.05 \times 10^{-3} \mathrm{~mm}^{2} / \mathrm{s}$, respectively. Regarding the MD values of the posterior limb of the left internal capsule, the values were not statistically significant $(p=0.68)$ in patients compared to controls (Table 5). in ROC curve analysis, the AUC of the MD values of the frontal lobe, posterior limb of the internal capsule, and cerebellar hemisphere on the right side used to differentiate patients from controls were $0.8,0.904$, and 0.927 , respectively, and the cut-off points to differentiate both groups were $0.79,0.74$, and $0.73 \times 10^{-3} \mathrm{~mm}^{2} / \mathrm{s}$, while those of the left frontal lobe and left cerebellar hemisphere were 0.839 and 0.893 , respectively, with cut-off points of 0.76 and $0.75 \times 10^{-3} \mathrm{~mm}^{2} / \mathrm{s}$, respectively. On the right side, for the frontal lobe, the sensitivity, specificity, andpositive and negative predictive values were $60.6 \%, 93.3 \%, 95.2 \%$, and $51.9 \%$, respectively, with an accuracy of $70.8 \%$. For the posterior limb internal capsule, the sensitivity, specificity, and positive and negative predictive values were $84.8 \%$, $93.3 \%, 96.6 \%$, and $73.7 \%$, respectively, with an accuracy of $87.5 \%$. For the cerebellum the sensitivity, specificity, and positive and negative predictive values were $87.9 \%$, $86.7 \%, 93.5 \%$, and $76.5 \%$, respectively, with an accuracy of $87.5 \%$. On the left side, for the frontal lobe, the sensitivity, specificity, and positive and negative predictive values were $78.8 \%, 66.7 \%, 77.1 \%$, and $53.8 \%$, respectively, with an accuracy of $70.7 \%$. For the cerebellum the sensitivity, specificity, and positive and negative predictive values were $84.8 \%, 80 \%, 90.3 \%$, and $70.6 \%$, respectively, with an accuracy of $83.3 \%$ (Table 7 ).

\section{Correlation}

There was positive correlation between the disease duration and the measured MD values of the right and left hippocampal head $(r=0.37, p=0.036)$.

Table 6. Results of receiver operating characteristic (ROC) curve of fractional anisotropy (FA) of the selected white matter regions with calculation of cut-off values, area under the curve (AUC), accuracy, sensitivity, and specificity in differentiating patients and controls

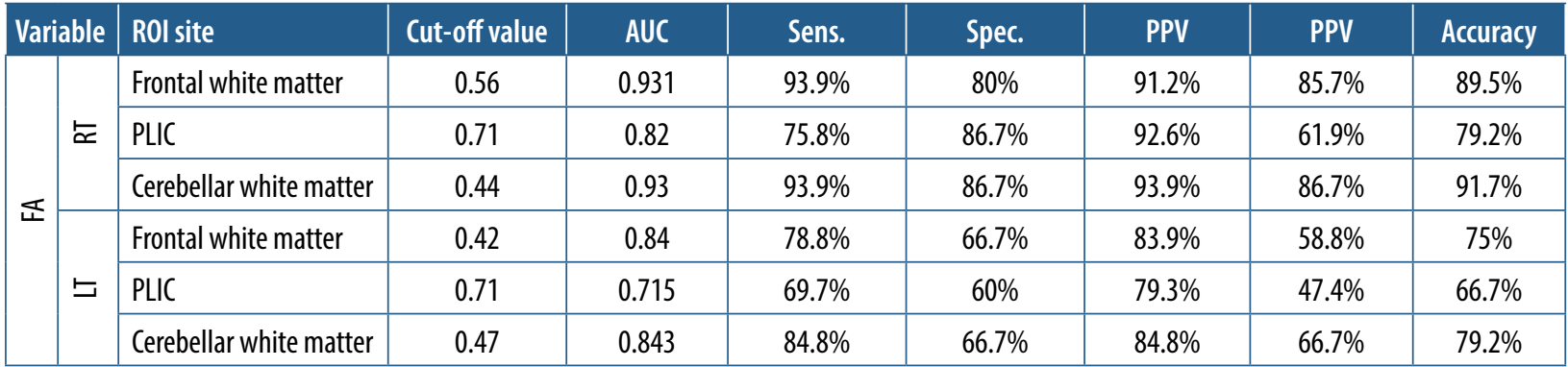

Table 7. Results of receiver operating characteristic (ROC) curve of mean diffusivity (MD) of the selected white matter regions with calculation of cut-off values, area under the curve (AUC), accuracy, sensitivity, and specificity in differentiating patients and controls

\begin{tabular}{|c|c|c|c|c|c|c|c|c|c|}
\hline \multicolumn{2}{|c|}{ Variable } & ROI site & Cut-off value & AUC & Sens. & Spec. & PPV & PPV & Accuracy \\
\hline \multirow{6}{*}{$\sum$} & \multirow{3}{*}{$\bar{x}$} & Frontal white matter & 0.79 & 0.8 & $60.6 \%$ & $93.3 \%$ & $95.2 \%$ & $51.9 \%$ & $70.8 \%$ \\
\hline & & PLIC & 0.74 & 0.904 & $84.8 \%$ & $93.3 \%$ & $96.6 \%$ & $73.7 \%$ & $87.5 \%$ \\
\hline & & Cerebellar white matter & 0.73 & 0.927 & $87.9 \%$ & $86.7 \%$ & $93.5 \%$ & $76.5 \%$ & $87.5 \%$ \\
\hline & \multirow{3}{*}{ 上 } & Frontal white matter & 0.76 & 0.839 & $78.8 \%$ & $66.7 \%$ & $77.1 \%$ & $53.8 \%$ & $70.7 \%$ \\
\hline & & PLIC & 0.72 & 0.527 & $66.7 \%$ & $46.7 \%$ & $73.3 \%$ & $38.9 \%$ & $60.4 \%$ \\
\hline & & Cerebellar white matter & 0.75 & 0.893 & $84.8 \%$ & $80 \%$ & $90.3 \%$ & $70.6 \%$ & $83.3 \%$ \\
\hline
\end{tabular}




\section{Discussion}

Migraine is a neurological disorder characterized by headache that occurs either unilaterally or bilaterally with duration ranging from hours to days [18]. Although it is common, its pathogenesis is not yet fully understood [19]. However, many investigations of neurotransmitters, neuromodulators, and functional neuroimaging studies have helped us to know the morphological and physiological changes occurring during migraine attacks [20]. PET studies have shown important brain changes in migraine, but with limited anatomical details [21]. There are any theories to explain the pathogenesis of migraine, like cortical spreading depression and hyperexcitability of cerebral cortex resulting in different brain microstructure changes [22].

Many of the studies done to detect grey and white matter microstructural changes in migraineurs enrolled mixed groups of patients - those having migraine with aura together with those having migraine without aura. This resulted in inconsistent and contradictory results due to sample heterogeneity [2], so in our study we enrolled a homogenous group containing 33 patients all having migraine without aura.

In our study, the highest migraine headache prevalence was detected between the ages of 20 and 40 years, with a high female predominance (female-to-male ratio $5.6: 1$ ). Similar results were reported in a study by Kandil et al. in 2016 [11] in the Assiut district of Egypt, which indicated that the highest prevalence of migraine was at the ages 20-40 years. Also, we found a wide range of disease duration in the patient group (1-24 years). The patient group consisted of only 33 subjects, which could be related to the small number of cases that met the inclusion criteria during the limited study duration. This study was just the first step in this issue, and we are preparing to apply the study on a large number of cases with multi-centre cooperation in the future. This large sample volume will give a greater chance for the appearance of other disease factors affecting the brain structures to become prominent.

In our study we also fund that the female-to-male ratio was $2.4: 1$. The difference in the male-to-female ratio may be due to either the small sample size of our study compared to their study or due to the inhomogeneity of the sample enrolled in the study of Kandil et al., because they enrolled all types of migraine while in our study only migraine patients without aura were studied.

Functional and structural differences of the cerebral white and grey matter were detected between migraineurs and healthy controls by several MRI studies [3,4]. Many studies were performed to identify the different affected grey and white matter regions in migraineurs; these regions included the frontal and parietal lobes, basal ganglia, brainstem, limbic system, and cerebellum [15,23]. In the current study we used 3 white matter regions (frontal lobe, posterior limb internal capsule, and cerebellum) and 3 grey matter regions (thalamus, globus pallidus, and hippocam- pus head) on each side, so the total number involved in our study was 6 grey matter and 6 white matter regions.

DTI has become an established tool in the investigation of central nervous system tissue microstructure and anatomy. DTI gives quantitative data on the motion of the molecular water. MD and FA are the most clinically used parameters. MD determines the magnitude of diffusion, while FA reveals the degree of diffusion and anisotropy $[15,24]$. Recent studies revealed the presence of microstructural changes in migraineurs, which are not seen in normal appearing brain tissues on conventional MRI $[2,25,26]$. In light of the above-mentioned neuroimaging studies, we thought that we could provide additional evidence of microstructural changes in patients having migraine without aura by obtaining the MD and FA measurements in the mentioned grey and white matter regions and comparing the results with those of healthy subjects, as well as to correlate the acquired data with the disease duration, attack frequency, and disease laterality.

In our study all the included patients $(n=33)$ had normal appearing brain tissue on conventional MRI in spite of the DTI changes, which can be due to the repeated mild ischaemic insults during migraine attacks, which may cause a loss in the structural barriers of molecular motion of water that can be detected by DTI and not detected by conventional MRI pulse sequences like T2WI and FLAIR [25]. This could be because the damage was below the minimum level that can be detected by conventional MRI; also, brain resistance mechanisms can be strong enough to prevent the appearance of these changes on routine MRI [27].

On DTI done for all patient and control groups involved in our study, we found a statistically significant difference in the MD values of the selected grey matter regions on the right side and the selected white matter regions on both sides except the posterior limb of the left internal capsule, and in FA values of both frontal lobes together with the selected grey matter regions on the right side and left hippocampal head of the patient group compared to controls. These changes could be detected instead of the normal conventional MRI examinations done for all patients, and this is attributed to the fact that DTI can detect tissue microstructural changes occurring in different pathologies that could not be detected by conventional MRI; this is because DTI parameters (MD and FA) become abnormal before the appearance of the morphological changes on conventional MRI. DTI also improved the sensitivity and objectivity in early detection of minor developmental and long-term changes along specific anatomical pathways that could be missed by the relatively subjective conventional MRI [28-30].

The mentioned changes in DTI parameters resulting from cell death could be explained by the migraine theories. First, the cortical spreading depression induces neurotransmitters leading to neuroinflammation and stimulation of glial cells [31]; moreover, it alters the brain 
circulation making it more liable to ischaemia and hence cell death resulting in different grey and white matter microstructural changes [32]. The other theory of hyperexcitability of the cerebral cortex may also play a role in the microstructural alterations seen in migraine $[22,33]$. Welch et al. reported that there is non-haeme iron deposition in the ganglionic region and brain stem in cases of recurrent migraine by repeated activation of the pain system, which represent cellular injury [20].

MD is sensitive to changes in the grey matter; this could be attributed to the mainly isotropic nature of diffusion in the grey matter. On the other hand, FA is sensitive to white matter changes; this is related to the anisotropic nature of diffusion in the white matter together with the high sensitivity of FA to the low values of diffusion anisotropy. In the current study, regarding the significant high MD values seen in the majority of the selected white matter regions, this could be explained by the cell death with subsequent increased extracellular spaces making diffusion freer [34]. While the significantly low FA values in some of the selected grey matter regions, mainly on the right side, could be related to the presence of white matter within some of the grey matter structures like the thalamus, which is formed of grey matter nuclei separated by white matter, so any changes in its white matter component could be detected through abnormal FA $[35,36]$.

In our study we found a discrepancy in the FA and $M D$ values in the same selected areas. This could be explained by that FA and MD may vary independently because the damaged brain has glial and neuronal cells; therefore, they have sufficient cell density to prevent the effect on MD, while, because of the disorganization, the FA is reduced. Added to that, microglial and astrocyte activation leads to reactive gliosis preventing the effect of cell death on DTI parameters [34].

In our study, statically significant differences were detected in MD and FA values of the selected right-sided grey and white matter regions compared to those on the left side. This could be explained by the dominance of the right basal ganglionic and limbic system structures in controlling the autonomic activity, which is usually involved during migraine attacks, so further studies are needed to correlate imaging findings with autonomic dysfunction [4].

Significant positive correlation could be found between the MD values of the right hippocampal head and disease duration $(r=0.37, p=0.036)$. This can be related to the fact that the longer the disease duration, the greater the frequency of attacks, leading to more susceptibility of brain tissue to ischaemia and as a result more cell death, resulting in increased $M D$ values.

\section{Limitation}

The limitations of this study are the small number of patients enrolled in the study and the absence of follow-up DTI for the patients, so studies on larger numbers should be done together with serial follow-up DTI studies. Also, it is better to do further studies using higher tesla scanners like 3 Tesla, with the application of advanced postprocessing DTI and diffusion Kurtosis, which could give better results than our study done using a 1.5 Tesla scanner. Also, the side of the brain region affected should be considered and whether they have different rules in migraine disorders.

\section{Conclusions}

DTI can detect microstructural changes of the grey and white matter in patients having migraine without aura, which cannot be detected by conventional MRI.

\section{Conflicts of interest}

The authors report no conflict of interest.
1. Headache Classification Committee of the International Headache Society (IHS). The international classification of headache disorders, $3^{\text {rd }}$ edition (beta version). Cephalalgia 2013; 33: 629-808.

2. Zhang J, Yi-Lan Wu, Jingjing Su, et al. Assessment of gray and white matter structural alterations in migraineurs without aura. J Headache Pain 2017; $18: 74$.

3. Maleki N, Gollub RL. What have we learned from brain functional connectivity studies in migraine headache? Headache 2016; 56: 453-461.

4. Goadsby PJ, Holland PR, Martins-Oliveira M, et al. Pathophysiology of migraine: a disorder of sensory processing. Physiol Rev 2017; 97: 553-622.

5. Chen Z, Chen X, Liu M, et al. Texture features of periaqueductal gray in the patients with medication-overuse headache. J Headache Pain 2017; 18: 14.
6. Iwasaki A, Suzuki K, Takekawa H, et al. The relationship between right-to-left shunt and brain white matter lesions in Japanese patients with migraine: a single center study. J Headache Pain 2017; $18: 3$.

7. Chen Z, Chen X, Liu M, et al. Disrupted functional connectivity of periaqueductal gray subregions in episodic migraine. J Headache Pain 2017; 18: 36.

8. Chen Z, Chen X, Liu M, et al. Altered functional connectivity architecture of the brain in medication overuse headache using resting state fMRI. J Headache Pain 2017; 18: 25.

9. Jia Z, Yu S. Grey matter alterations in migraine. A systematic review and meta-analysis. Neuroimage Clin 2017; 14: 130-140.

10. El-Sherbiny NA, Masoud M, Shalaby NM, Shehata HS. Prevalence of primary headache disorders in Fayoum Governorate, Egypt. J Headache Pain 2015; 16: 85. 
11. Kandil MR, Hamed SA, Fadel KA, et al. Migraine in Assiut Governorate, Egypt: epidemiology, risk factors, comorbid conditions and predictors of change from episodic to chronic migraine. Neurol Res 2016; 38: 232-241.

12. Zhang Y, Parikh A, Qian S. Migraine and stroke. Stroke Vasc Neurol 2017; 2: 160-167.

13. Luijkx T, Gaillard F. Migraine. Available from: https://radiopaedia. org/articles/migraine.2019.

14. Coppola G, Tinelli E, Lepre C, et al. Dynamic changes in thalamic microstructure of migraine without aura patients: a diffusion tensor magnetic resonance imaging study. Eur J Neurol 2014; 21: 287-292.

15. Kara B, Kiyat Atamer A, Onat L, et al. DTI findings during spontaneous migraine attacks. Clin Neuroradiol 2013; 23: 31-36.

16. Chong CD, Todd J, Schwedt JT. Migraine affects white-matter tract integrity: a diffusion-tensor imaging study. Cephalalgia 2015; 35: 1162-1171.

17. Headache Classification Committee of the International Headache Society (IHS) The International Classification of Headache Disorders, $3^{\text {rd }}$ edition. Cephalalgia 2018; 38: 1-211.

18. Cutrer FM. Pathophysiology of migraine. Semin Neurol 2010; 30 : 120-130.

19. Lipton RB, Bigal ME. Migraine: epidemiology, impact, and risk factors for progression. Headache 2005; 45 Suppl 1: S3-13.

20. Welch KM, Nagesh V, Aurora SK, Gelman N. Periaqueductal gray matter dysfunction in migraine: cause or the burden of illness? Headache 2001; 41: 629-637.

21. Denuelle M, Fabre N, Payoux P, et al. Hypothalamic activation in spontaneous migraine attacks. Headache 2007; 47: 1418-1426.

22. Pierelli F, Iacovelli E, Bracaglia M, et al. Abnormal sensorimotor plasticity in migraine without aura patients. Pain 2013; 154: 1738-1742.

23. Zhihua J, Shengyuan Yu. Grey matter alterations in migraine: a systematic review and meta-analysis. Neuroimage Clin 2017; 14: 130-140.

24. Mukherjee P, Berman JI, Chung SW, et al. Diffusion tensor MR imaging and fiber tractography: theoretic underpinnings. Am J Neuroradiol 2008; 29: 632-641.

25. Rocca MA, Colombo B, Inglese M, et al. A diffusion tensor magnetic resonance imaging study of brain tissue from patients with migraine. J Neurol Neurosurg Psychiatry 2003; 74: 501-503.
26. Yu D, Yuan K, Zhao L, et al. White matter integrity affected by depressive symptoms in migraine without aura: a tract-based spatial statistics study. NMR Biomed 2013; 26: 1103-1112.

27. Wisnowski JL, Panigrahy A, Painter MJ, Watchko JF. Magnetic resonance imaging of bilirubin encephalopathy: current limitations and future promise. Semin Perinatol 2014; 38: 422-428.

28. Wang X, Wu W, Hou BL, et al. Studying neonatal bilirubin encephalopathy with conventional MRI, MRS, and DWI. Neuroradiology 2008; 50: 885-893.

29. Razek AA, Nada N. Correlation of choline/creatine and apparent diffusion coefficient values with the prognostic parameters of head and neck squamous cell carcinoma. NMR Biomed 2016; 29: 483-489.

30. Abdel Razek AAK. Arterial spin labelling and diffusion-weighted magnetic resonance imaging in differentiation of recurrent head and neck cancer from post-radiation changes. J Laryngol Otol 2018; 132: 923-928.

31. Charles A, Brennan K. Cortical spreading depression - new insights and persistent questions. Cephalalgia 2009; 29: 1115-1124.

32. Ayata C, Lauritzen M. Spreading depression, spreading depolarizations, and the cerebral vasculature. Physiol Rev 2015; 95: 953-993.

33. Chadaide Z, Arlt S, Antal A, et al. Transcranial direct current stimulation reveals inhibitory deficiency in migraine. Cephalalgia 2007; 27: 833-839.

34. Razek AAKA, El-Serougy L, Abdelsalam M, et al. Differentiation of residual/recurrent gliomas from postradiation necrosis with arterial spin labeling and diffusion tensor magnetic resonance imagingderived metrics. Neuroradiology 2018; 60: 169-177.

35. Sedrak M, Gorgulho A, Frew A, et al. Diffusion tensor imaging and colored fractional anisotropy mapping of the ventralis intermedius nucleus of the thalamus. Neurosurgery 2011; 69: 1124-1129.

36. Mang S, Busza A, Reiterer S, et al. Thalamus segmentation based on the local diffusion direction: a group study. Magn Reson Med 2012; 67: 118-126. 